\title{
PERFIL E QUALIDADE DE VIDA DA EQUIPE ASSISTENCIAL DE ENFERMAGEM
}

\author{
PROFILE AND QUALITY OF LIFE OF THE NURSING ASSISTANT TEAM
}

\author{
Camila Zanesco, Julyane Felipette Lima, Aline Bissolotti, Silvia Silva de Souza, Débora Tavares \\ Resende e Silva
}

\author{
Universidade Federal da Fronteira Sul - UFFS
}

\begin{abstract}
Objective (s) of the study: to evaluate the sociodemographic profile and the quality of life of the nursing team of a reference service in nephrology, private and agreed to the single health system. Methodology: exploratory, transversal, quantitative study. Data collection took place in July, August and September 2016, using the sociodemographic questionnaire and the Short Form-36 instrument to collect information. Results: Our data showed that all participants have a single job, and the working hours are allocated in day shifts. Most aresatisfied with current employment (95.25\%), and with remuneration (61.91\%). The evaluated participants had an increased general mean of functional domains (89.76), limitations due to emotional problems $(87,30)$ and Limitations in social activities (80.95), and the lowest general average was in general health status 62.86). Conclusion: The quality of life of the nursing team is essential for an adequate treatment, since this team is responsible for a part of the treatment of the patients directly connected to it, being important to check the general health of the same so that the treatment be as effective as possible.
\end{abstract}

Key words: Nursing team; Quality of life; Nephrology

\section{Resumo}

Objetivo(s) do estudo: avaliar o perfil sociodemográfico e a qualidade de vida da equipe de enfermagem de um serviço referência em nefrologia, privado e conveniado ao sistema único de saúde. Metodologia: estudo de caráter exploratório, transversal, quantitativo. A coleta de dadosocorreu em julho, agosto e setembro de 2016, sendo utilizado o questionário sociodemográfico e o instrumento Short Form-36 para coleta das informações. Resultados: Nossos dados mostraram que todos participantes possuem um único emprego, e, os horários de trabalho são alocados em turnos diurnos. A maioria está satisfeita com o emprego atual (95,25\%), e com a remuneração (61,91\%).As participantes avaliadas apresentaram média geral aumentadados domínios Capacidade funcional $(89,76)$, Limitações por problemas emocionais $(87,30)$ e Limitações em atividades sociais $(80,95)$, e a menor média geral foi no aspecto estado geral de saúde $(62,86)$. Conclusão: A qualidade de vida da equipe de enfermagem é essencial para um tratamento adequado, visto que é esta equipe a responsável por uma parte do tratamento dos pacientes à ela diretamente ligados, sendo importante verificar o estado geral de saúde dos mesmos para que o tratamento seja o mais eficaz possível.

Palavras chave: Equipe de enfermagem; Qualidade de vida; Nefrologia. 


\section{Introdução}

A Qualidade de Vida (QV) contempla uma ampla gama de questões, como: nível de escolaridade, condições de saneamento básico, acesso a serviços de saúde, satisfação em relação as condições de saúde, emocionais, de trabalho e outras. O trabalho compõe o agrupamento das necessidades humanas básicas, e constitui-se como um dos determinantes com maior influência sobre a QV. Condições indesejadas e insatisfação no ambiente de trabalho acarretam em alterações físicas e emocionais, implicando negativamente na QV dos indivíduos ${ }^{1-5}$.

Devido a magnitude e influência em relação a QV em diversos aspectos da vida do indivíduo, tem crescido o números de estudos que ponderem a $Q V$, enfatizando o binômio "trabalho-saúde". Quando considerados os profissionais é importante considerar o contexto do trabalho e fora dele, sendo conhecido que fatores geradores de incômodo externos ao ambiente de trabalho afetam o desenvolvimento e rendimento no ambiente laboral e contrariamente o mesmo pode ocorre ${ }^{3,5-6}$.

Trabalhar em prol da saúde e bem estar do próximo, da coletividade, convivendo com a dor, sofrimento e morte, são questões que contribuem para o surgimento de sofrimento psicológico e afetam diretamente a atuação profissional. Sendo os profissionais de enfermagem suscetível a estas questões, é imprescindível a avaliação da QV, para a partir dos resultados se necessário implementar medidas de mediação e ou prevenção, para que assim o trabalho seja executado de forma ideal ${ }^{3}$.

Partindo do exposto, observou-se escassez de estudos na área de avaliação de QV de equipes de saúde em seus locais de trabalho. Sendo assim, este estudo objetivou avaliar o perfil sociodemográfico e a $\mathrm{QV}$ da equipe de enfermagem de um serviço de atendimento a doentes renais crônicos com oferta de hemodiálise.

\section{Metodologia}

A pesquisa possui caráter exploratório, transversal, com análise quantitativa, e avaliou o perfil sociodemográfico e a qualidade de vida (QV) da equipe de enfermagem de um serviço que atende doentes renais crônicos com oferta de hemodiálise (HD) no oeste catarinense. 0 período da coleta de dados ocorreu entre julho, agosto e setembro de 2016.

O serviço onde foi realizado o estudo localiza-se no município de Chapecó estado de Santa Catarina, possui cerca de 183.530 habitantes ${ }^{7}$, sendo referência para inúmeros municípios de menor porte da região. Dentre as modalidades de terapia renal substitutiva (TRS), são ofertadas no referido serviço: HD e transplante renal. Possui taxa de ocupação de 216 (73,15\%), o mesmo compõe o percentual de $48 \%$ de serviços que ofertam TRS e localizam-se fora do ambiente hospitalar ${ }^{8}$.

A amostragem foi transversal e foram incluídos na pesquisa todos os membros da equipe de enfermagem. Foram distribuídos os questionários para 3 enfermeiras e 27 técnicos de enfermagem. Foi realizado um momento de explicação da pesquisa e justificativas para a mesma, e, os que aceitaram participar devolveram 0 instrumento preenchido. 0 instrumento possuía duas etapas: etapa 1 : questionário sociodemográfico; etapa 2: questionário de QV: Medical Outcomes Study 36 - Item Short - Form Health Survey (SF-36).

O SF-36 é um instrumento de coleta de dados genérico que avalia a QV. O SF-36 é de fácil administração e compreensão, compreendendo perguntas curtas e respostas objetivas $^{9}$. Possui 36 questões divididas em domínios: 1) físico (avalia-se a dor, fadiga, náuseas, efeitos da medicação, entre outros), 2) funcional (aborda-se as atividades diárias e desempenho no trabalho), 3) psicológico (satisfação com seu estado de saúde, com a vida, e sintomas gerais de depressão e ou ansiedade), 4) social (contempla aspectos relacionados a família, profissional e social) ${ }^{1}$. A partir dos domínios são apontadas as oito dimensões: 1) capacidade funcional, 2) aspectos físicos, 3) dor, 4) estado geral de saúde, 5) vitalidade, 6) aspectos sociais, 7) aspectos emocionais, 8) saúde mental e uma questão comparativa da condição de saúde atual e a de um ano atrás. Apresenta um escore final de 0 (zero) a 100 (cem) - obtido por meio de cálculo do Raw Scale, onde o zero corresponde ao pior estado geral de saúde e o 100 corresponde ao melhor estado de saúde. Com a obtenção das respostas, as mesmas são substituídas por um escore, e posteriormente o mesmo é transformado em uma escala de 0 a $100^{1}$.

Os dados foram coletados e armazenados em planilha no Libre Office Calc, analisados por meio do mesmo programa, onde se calculou as frequências absolutas (n) e relativas (\%) para as variáveis categóricas. Após agrupar as respostas em domínios do questionário SF-36, foi utilizada a padronização dos escores atribuídos às respostas, baseado no instrumento, o qual traz a 
forma de cálculo de cada ponto em questão, utilizando o programa Libre Office Calc ${ }^{9}$.A pesquisa foi aprovada pelo Comitê de Ética em Pesquisa da UFFS, sob o CAAE: 49503215.4.0000.5564. Número do Parecer: 1.381.525/UFFS/2015.

\section{Resultados e Discussões}

\section{Perfil sociodemográfico}

A equipe de enfermagem é composta por 30 (100\%) profissionais sendo que desses 21 (70\%) participaram da pesquisa, e $100 \%$ dos participantes da pesquisa eram do sexo feminino. Quanto a formação/categoria profissional dos 21 participantes $14,29 \%(n=3)$ eram enfermeiras e $85,71 \%(n=18)$ técnicas de enfermagem.

Em relação a idade observou-se que 6 participantes $(28,57 \%)$ tinham entre $21-30$ anos, $10(47,62 \%)$ entre $31-40$ anos, 3 (14,29\%) entre 41-50 anos e 2 (9,52\%) entre 51-60 anos. Em relação ao estado civil 9 participantes $(42,86 \%)$ são solteiros, 6 (28,58\%) casados, 3 (14,29\%) divorciados e $3(14,29 \%)$ estão em uma união estável; um total de 57,14\% têm filhos.

Considerando a existência de doença prévia entre os membros da equipe, observou-se que 3 $(14,29 \%)$ possuem algum tipo de doença (hipertensão arterial; hipertireoidismo; espondilite anquilosante) e 7 das participantes $(33,33 \%)$ fazem uso de algum medicamento (anticoncepcional; alpharzan; puran; sulfa; losartana). Considerando a religião 18 (85,71\%) são católicos e $3(14,29 \%)$ são evangélicos. O município sede do serviço também é onde $100 \%$ dos entrevistados residem.

No sentido de identificar as rotinas e o conhecimento/preparação que receberam durante a formação em relação a doenças crônicas e a doença renal crônica (DRC) especificamente, satisfação com as atividades que desenvolve e com a remuneração, foram aplicadas algumas questões. Para 18 participantes $(85,1 \%)$ ocorreu preparação durante a formação para lidar com doenças crônicas e DRC em específico, apenas 3 (14,29\%) não tiveram nenhum contato com esse assunto. Em relação a orientações durante a formação sobre questões que envolvem a HD, 15 (71,43\%) disseram que tiveram e $6(28,57 \%)$ disseram que não.

Considerando o turno em que trabalham 13 participantes $(61,90 \%)$ são alocados no primeiro turno e $8(38,10 \%)$ no segundo turno. Quando questionados sobre a satisfação com o emprego atual observou-se que 95,25\% afirmaram que estão satisfeitos. Em relação a remuneração 13 participantes $(61,91 \%)$ confirmaram a satisfação, 6 (28,57\%) não estão e 2 (9,52\%) não responderam. As participantes não possuem outro emprego. Quando questionadas sobre a prática de atividade física, 11 (52\%) disseram praticar atividades físicas.

\section{Qualidade de vida (QV)}

Considerando a QV a partir do instrumento SF-36 neste estudo observou-se que as participantes avaliadas apresentaram média geral aumentada dos domínios Capacidade funcional $(89,76)$, Limitações por problemas emocionais $(87,30)$ e Limitações em atividades sociais $(80,95)$, e a menor média geral foi no aspecto estado geral de saúde $(62,86)$ (Tabela 1$)$.

Tabela 1. Pontuação obtida nos oito domínios do questionário SF-36 nas avaliações com a equipe de enfermagem. $\mathrm{N}=21$.

\begin{tabular}{l|c|c}
\hline \multicolumn{1}{c|}{ Domínio } & Média & $\begin{array}{c}\text { Número de } \\
\text { Pessoas }\end{array}$ \\
\hline Capacidade Funcional & 89,76 & 21 \\
\hline $\begin{array}{l}\text { Limitações por aspectos } \\
\text { físicos }\end{array}$ & 76,19 & 21 \\
\hline Dor & 74,40 & 21 \\
\hline Estado geral da saúde & 62,86 & 21 \\
\hline Saúde mental & 64,95 & 21 \\
\hline $\begin{array}{l}\text { Limitações por } \\
\text { problemas emocionais }\end{array}$ & 87,30 & 21 \\
\hline $\begin{array}{l}\text { Limitações em } \\
\text { atividades sociais }\end{array}$ & 80,95 & 21 \\
\hline \begin{tabular}{l} 
Vitalidade \\
\hline
\end{tabular} & 65,00 & 21 \\
\hline
\end{tabular}

Fonte: Dados da pesquisa.

O presente estudo foi realizado com o objetivo de avaliar o perfil sociodemográfico e a qualidade de vida (QV) de uma equipe de enfermagem em um serviço de nefrologia do oeste catarinense. A QV como visto ultrapassa a ausência ou presença de saúde, é diretamente ligada a questões envoltas a educação, saneamento básico, acesso a serviços de saúde, satisfação e condições de trabalho, e outras questões ${ }^{1}$.

Os dados demonstraram prevalência de mulheres na equipe de enfermagem, assim como visto em outros estudos o número de indivíduos do sexo masculino dentre os trabalhadores na área da enfermagem é reduzido, um dos estudos apresentou cerca de $86,82 \%$ de 129 trabalhadores ${ }^{10}$ e $71 \%$ de 38 trabalhadores ${ }^{6}$ eram mulheres, por último ${ }^{11}$, em um estudo realizado em um serviço de nefrologia onde $100 \%$ de 12 trabalhadores eram do sexo feminino ${ }^{11}$. Este 
achado remete suma importância, visto que é conhecida a relação direta entre o sexo feminino e desgastes físicos e emocionais ${ }^{6}$. Outro importante aspecto com influência direta sobre a $\mathrm{QV}$, consiste na segurança proporcionada pelo ambiente, qualidade dos materiais a serem utilizados e existência de protocolos que respaldem a ação do profissional.

A prevalência de idade foi para a faixa etária entre 31 e 40 anos; o estado civil solteira englobou mais profissionais, diferindo de estudos publicados, onde o predomínio foi de casadas ${ }^{6}$. A maioria das profissionais informou ter filhos $57,14 \%$,os dados exposto remetem a associação do desgaste físico e emocional ligadas a dedicação necessária no ambiente de trabalho, às atividades domésticas, e à família. Em contrapartida as relações sociais, a impressão de pertencimento a um determinado grupo, constituem-se como um aporte, contribuindo positivamente para melhores níveis de QV de profissionais atuantes na área da saúde ${ }^{12}$. Considerando que o trabalho tem para a grande maioria dos indivíduos lugar central, sendo inseparável da vida humana, o mesmo consiste no principal determinante da $\mathrm{QV}^{1}$; aplicamos as questões no sentido de reconhecer a percepção perante a satisfação da equipe com o trabalho e remuneração, sendo que os resultados mostraram-se positivos. Quando questionados sobre a satisfação ou não com o trabalho atual, $95,24 \%$ dos entrevistados responderam positivamente a questão, fator primordial empregado a permanência no ambiente de trabalho ${ }^{11}$. Adiante o trabalho constitui-se como maior determinante nos níveis de $\mathrm{QV}^{1}$, onerando a necessidade do profissional estar satisfeito com este. O contentamento e envolvimento com o trabalho, culminam em melhor produtividade por parte do funcionário ${ }^{1,12}$.

A alocação diurna dos turnos de trabalho utilizada no referido serviço, estabelece um importante fator na avaliação positiva encontrada. O desequilíbrio imposto em relação a comunidade quando se cumpre rotinas noturnas relacionadas ao trabalho é evidente, ademais situações de sonolência, insônia e outras, podem surgir ao longo do dia ${ }^{1}$.

Dos entrevistados $100 \%$ não possuem outro vínculo empregatício, estar em apenas um emprego cuja remuneração supre as necessidades econômicas, pode estar imbricada neste caso ao caráter privado do serviço investigado. A dispensação de atividades em um único serviço relaciona-se a menor frequência de desgastes a nível emocional e físico, e maior tempo disponível para desempenhar atividades relacionadas ao próprio bem-estar. Em relação a prática de atividade física ocorreu divergência em relação ao exposto em estudo prévio, o qual encontrou prevalência de sedentarismo em profissionais da saúde na proporção de $27,5 \%$ para 3.347 entrevistados ${ }^{13}$.

A difusão da prática de atividades físicas entre profissionais da área da saúde englobando a região Sul do País em cidades com menos de 200 mil habitantes é menos intensa,, até $6 \%$ menor em relação a cidades com população entre 200 a 500 mil habitantes ${ }^{13}$. A região Sul associou-se com maiores índices de sedentarismo considerando o público em questão, e no geral o público feminino apresentou-se mais ativo que o masculino, a influência para aderir uma rotina com atividades físicas tem a ver com os fatores sócio econômicos, carga horária semanal dispensada no ambiente de trabalho, número de empregos, ocupação/tipo de atividade profissional.

A prática de atividades físicas beneficia as rotinas no ambiente de trabalho e pessoal, o sedentarismo e ou níveis insuficientes de prática destas, podem comprometer a saúde do trabalhador, impactando negativamente na prestação de serviços à comunidade ${ }^{12}$.

Nesse sentido as pesquisas em torno da QV dos profissionais de saúde se intensificaram a partir dos anos 80, devido principalmente a atuação frente a condições vulneráveis a seu estado de saúde ${ }^{14}$.

São os profissionais de enfermagem que executam cerca de $60,00 \%$ das ações de atendimento aos clientes e os que estão fisicamente mais próximo dos usuários, sendo indispensável que os mesmos estejam bem, satisfeitos, inferindo boa QV, para que possam desempenhar as ações com eficiência ${ }^{14}$.

No que condiz como relacionamento entre profissional e paciente, o estabelecimento de uma boa relação constitui-se como fator de impacto positivo em prol do processo de cuidado ${ }^{15}$.

$\mathrm{Na}$ área da saúde cada profissão comporta suas peculiaridades a enfermagem não é diferente, dentre as quais podem ser destacadas: - quantitativo pessoal, formação técnica heterogênea, organização e a divisão de trabalho, predominância do sexo feminino, remuneração, os turnos e a constante vivência de tensões, todos esses aspectos e outros interferem na percepção de satisfação ou não da equipe de enfermagem com 0 trabalho desempenhado ${ }^{14}$.

A múltipla disponibilidade de campos de atuação para profissionais de enfermagem possui 
divergência perante as exigências físicas, psicológicas e de conhecimento dispensadas. Um estudo realizado em $2006^{1}$, que investigou a QV de uma equipe de enfermagem em uma central de materiais hospitalar, os menores índices forma para a dimensão da Dor, podendo decorrer da maior exigência quanto aos esforços físicos, discrepante das exigências quanto aos serviços empregado em um ambiente onde se realiza HD. Considerando todo o contexto histórico e as mudanças frequentes no ambiente de trabalho, relacionando-as com a capacidade humana de adaptar-se, pode-se levar a questões como: insatisfação generalizada, tédio, angústias, ambiguidades, ansiedade, despersonalização, frustração, alienação no trabalho, entre outras ${ }^{1}$. No trabalho são demandadas questões físicas, emocionais e mentais, podendo gerar comprometimento da QV e capacidade no trabalho ${ }^{10}$.

A QV ultrapassa a mera ausência de doenças, a complexidade deste parâmetro transpassa os limites dos aspectos físicos, como exposto o trabalho é o principal contribuinte para os níveis de QV, alterando principalmente os domínios: físico, psicológico e social ${ }^{16}$.

Resultados expressos em estudo prévio ${ }^{11}$ demonstram que a rotina no ambiente de trabalho é modulada por questões simples, como, o reconhecimento por parte dos pacientes, demonstrações de carinho, convivência harmoniosa entre os membros da equipe, entre outras, as quais estimulam o indivíduo a desempenhar suas ações.

\section{Conclusões}

A qualidade de vida da equipe de enfermagem é essencial para um tratamento adequado, visto que é esta equipe a responsável por uma parte do tratamento dos pacientes à ela diretamente ligados, sendo importante verificar o estado geral de saúde dos mesmos para que o tratamento seja o mais eficaz possível.

Apontamos a escassez de estudos com equipes de enfermagem em nefrologia dificultou comparações e impossibilitou o uso de referencial teórico com a mesma especificidade. Porém, destacamos a receptividade no serviço por parte da equipe de enfermagem e profissionais médicos, bem como da aceitação por parte dos acometidos de participação. Questões simples que facilitam e resultam positivamente no contexto geral da pesquisa.

\section{Referências}

1. Talhaferro B, Barboza DB,Domingos NAM. Qualidade devida da equipe de enfermagem da central de materiais e esterilização. Rer. Cie. Med. [revista online] 2006 [acesso em 10 de fevereiro de 2017];6 (15). Disponível em: http://periodicos.puccampinas.edu.br/seer/index.php/cienciasmedica s/article/viewFile/1079/1055

2. Santana SS, Fontanelle T, Magalhães LM. Assistência de enfermagem prestada aos pacientes em tratamento hemodialítico nas unidades de nefrologia. Revista Científica do ITPAC,[revista online]; 2013 julho [acesso em 10 de abril de 2018];6:(3). Disponível em: https://assets.itpac.br/arquivos/Revista/63/5.pdf 3. Maciel MED, Oliveira FND. Qualidade de vida do profissional técnico deenfermagem: a realidade de um hospital filantrópico em Dourados-MS. Revista Psico. e Saúde [revista online] 2014 [acesso em 10 de abril de 2017]; 6 (1). Disponível em: http://pepsic.bvsalud.org/pdf/rpsaude/v6n1/v6n 1a11.pdf

4. Santana JCB, Fortes NDM, Monteiro CLDA, Carvalho IM, Leonardo LMU, Albuguergue GD. Assistência de enfermagem em um serviço de terapia renal substitutiva:implicações no processo do cuidar. Enfermagem Revista: pucminas. [revista online] 2012 [acesso em 10 de abril de 2017]; 15 (2): 162-178. Disponível em: http://periodicos.pucminas.br/index.php/enferm agemrevista/article/view/4082

5. PRESTES FC, BECK CLC, MAGNANO TSBdeS, SILVA RMda, TAVARES JP. Contexto de trabalho em um serviço de hemodiálise: avaliação dos trabalhadores de enfermagem. Texto Contexto Enferm, [revista online] 2015 JulSet [acesso em 10 de abril de 2018]; 24(3). Disponível em: http://www.scielo.br/pdf/tce/v24n3/pt_01040707-tce-24-03-00637.pdf

6. Motke MB, Franco GFP. Qualidade de vida em saúde da equipe de enfermagem da unidade de emergência de um hospital de grande porte do interior do Rio Grande do Sul. Revista Contexto \& Saúde. [revista online] 2003 [acesso em 10 de abril de 2017];; 5 (1). Disponível em: https://www.revistas.unijui.edu.br/index.php/co ntextoesaude/article/view/1314

7. IBGE (Org.). Instituto brasileiro de geografia e estatística: Chapecó. Informações completas. 2010 [acesso em 10 de abril de 2017];. Disponível em: <http://www.cidades.ibge.gov.br/xtras/perfil.ph 
p lang $=\&$ codmun $=420420 \&$ search $=$ santacatarina |chapeco|infograficos:-informacoes-completas>. 8. Sesso RC, Lopes AA, Thomé FS, Lugon JR, Watanabe $Y$, Santos DRD. Relatório do Censo Brasileiro de Diálise Crônica 2012. J. Bras. Nefrol. [revista online] 2014 [acesso em 10 de abril de 2017]; $36 \quad$ (1). Disponível em: http://www.scielo.br/scielo.php?pid=S01012800 2014000100048\&script=sci_abstract\&tlng=pt

9. Ware J.E., et al. MAP-R for Windows: Multitrait/Multi-Item Analysis Program-Revised User's Guide. Health Assessment Lab. Boston, MA. 1997. IN GANDEK, Barbara, M.S., et al. Psychometric Evaluation of the SF-36 ${ }^{\circledR}$ Health Survey in Medicare ManagedCare. HEALTH CARE FINANCING REVIEW. 2004; 25 (4).

10. Queiroz DLD. Qualidade de vida e capacidade para o trabalho dos profissionais de enfermagem de um hospital de grande porte de Dourados/MS [dissertação online]. Campo Grande: Universidade Católica Dom Bosco; 2012 [acesso em 10 de fevereiro 2017];.. Disponível em: http://site.ucdb.br/public/mddissertacoes/8222-qualidade-de-vida-e-

capacidade-para-o-trabalho-dos-profissionais-deenfermagem-de-um-hospital-de-grande-portede-dourados-ms.pdf

11. Prestes FC, Beck CLC, Silva RMD, Tavares JP, Camponogara S, Burg G. Prazer-sofrimento dos trabalhadores de enfermagem de um serviço de hemodiálise. Revista Gaúcha de enfermagem [revista online] 2010 [acesso em 08 de abril de 2017];.; $31 \quad$ (4). Disponível em: http://www.scielo.br/scielo.php?script=sci_artte $x t \& p i d=S 1983-14472010000400018$

12. Haika DAS, Oliveira PEAD, Sales LOS, Pereira ACA, Santa-Rosa TTDA, Macedo CCS, et al. Qualidade de vida, satisfação e esforço/recompensa no trabalho, transtornos psíquicos e níveis de atividade física entre trabalhadores da atenção primária à saúde. Revista de Aps. [revista online] 2013[acesso em 06 de fevereiro de 2017];16(3). Disponível em: https://aps.ufjf.emnuvens.com.br/aps/article/vie w/1549

13. Siqueira FCV, Nahas MV, Facchini LA, Piccini RX, Tomasi E, Thumé E, et al. Atividade física em profissionais de saúde do Sul e Nordeste Brasil. Cad. Saúde Pública. [revista online] 2009 [acesso em 10 de abril de 2017]; 25(9): Disponível em: http://www.scielo.br/scielo.php?script=sci_artte xt\&pid=S0102-311X2009000900006.

14. Almeida ANFD, Gurgel ERS, Silva SRD. Qualidade de vida dos profissionais de enfermagem de um centro cirúrgico. R. bras. Qual. Vida. [revista online] 2014 [acesso em 21 de fevereiro de 2017]; 6 (4): 216-222. Disponível em:

https://revistas.utfpr.edu.br/rbqv/article/view/1 942

15. Rodrigues TA, Botti NCL. Cuidar e o ser cuidado na hemodiálise. Acta paul. Enferm. [revista online] 2009 [acesso em 10 de abril de 2017];.; $22 \quad$ (1):. Disponpível em: www.scielo.br/scielo.php?script=sci_arttext\&pid =S0103-21002009000800015

16. Bracarense CF, Costa NDS, Duarte JMG, Ferreira MBG, Simões ALDA. Quality of life atwork: speech of Professional softhe Family Health Strategy. Escola Anna Nery - Revista de Enfermagem [revista online]. 2015 [acesso em 10 de abril de 2017];:; 19 (4). Disponível em: http://www.scielo.br/scielo.php?script=sci_artte xt\&pid=S1414-81452015000400542

\section{Endereço para Correspondência}

Universidade Federal da Fronteira Sul - UFFS

Av. Fernando Machado, 108E - Centro, Chapecó - SC CEP.: 89814-470

e-mail: camila_zanesco@hotmail.com

Recebido em 22/10/2017

Aprovado em 02/05/2018

Publicado em 11/05/2018 